\title{
Thermal-lens spectrometer for studying thermophysical properties of fullerenes
}

\author{
I. V. Mikheev ${ }^{1,2}$, L. O. Usoltseva ${ }^{1,2}$, D. A. Ivshukov ${ }^{1}$, D. S. Volkov ${ }^{1,2}$, M. V. Korobov ${ }^{1}$, M. A. Proskurnin ${ }^{1,2}$ \\ ${ }^{1}$ Chemistry Department, Lomonosov Moscow State University, Moscow, Russia \\ ${ }^{2}$ Analytical Centre of Lomonosov Moscow State University / Agilent Technologies Authorized Partner \\ Laboratory, Moscow, Russia \\ mikheev.ivan@gmail.com, usoltsevalilya@gmail.com,dima.iv@mail.ru,dmsvolkov@gmail.com, \\ mkorobov49@gmail.com,proskurnin@gmail.com
}

DOI 10.17586/2220-8054-2018-9-1-14-16

\begin{abstract}
A thermal-lens spectrometer implementing back-synchronized detection technique with a mode-mismatched optical scheme was constructed. Steady-state and transient signals of thermal-lens spectrometry are used to characterize concentration parameters of aqueous fullerene dispersions (AFDs) at the level of $10^{-7}-10^{-5} \mathrm{M}$ and to assess thermophysical properties of AFDs. The detection limits of fullerenes in AFDs are $100 \mathrm{nM}$ for $\mathrm{C}_{60}, 80 \mathrm{nM}$ for $\mathrm{C}_{70}$ and $\mathrm{C}_{78}-\mathrm{C}_{88}$, and $60 \mathrm{nM}$ for $\mathrm{Y} @ \mathrm{C}_{82}$, which are 20-fold lower than for spectrophotometry. Suitable precision of measurements of thermal diffusivity and thermal effusivity for AFDs is shown.
\end{abstract}

Keywords: thermal-lens spectrometry, pristine fullerenes, aqueous fullerene dispersions, thermal diffusivity.

Received: 21 June 2017

Revised: 10 September 2017

\section{Introduction}

Thermal-lens spectrometry (TLS) is used for the complex characterization of various materials $[1,2]$. This method is used for assessing the material optical parameters in UV/Vis/NIR governing or associated with the distribution of heat of different materials giving both thermal and optical properties in a single experiment $[1,3]$. In this paper, we constructed a thermal-lens setup to obtain the concentration parameters with high sensitivity and to estimate thermal diffusivity of aqueous fullerene dispersion (AFD) samples.

\section{Materials and methods}

The thermal-lens spectrometer (Fig. 1) uses a cw diode excitation laser working at $445 \mathrm{~nm}$ (power range in the sample $40-400 \mathrm{~mW}$ ), beam waist diameter $300 \pm 10 \mu \mathrm{m}$. Absorption of the excitation radiation by the sample induces refractive heterogeneity (the thermal-lens effect) causing defocusing of a TEM00 He-Ne laser probe beam (HRP020, ThorLabs, USA; $632.8 \mathrm{~nm}$; waist size $-25.0 \pm 0.2 \mu \mathrm{m}$; power $-4 \mathrm{~mW}$ ). The excitation beam goes through an electromechanical chopper, is focused with the lens L1 (focal length $330 \mathrm{~mm}$ ), goes through a dichroic mirror DM of ZR-100 type (Russia) and, next, to the sample. A part of excitation beam energy is reflected with the flat plate to the synchronizing photodiode (L-3DP3C, Panasonic, Japan), connected to the Control Unit. The probe beam is directed to the adjustment mirror using a system of a dichroic mirror and a focusing lens L2 (focal length $185 \mathrm{~mm}$ ). After the sample the excitation and probe beams are preliminarily separated with a dichroic mirror of ZR-100 type. The excitation beam is directed to Photometry PD to account for the photometric (transmission) signal of the excitation beam. Also, this mirror avoids the blooming of a thermal lens in the following bandpass filter. The excitation beam is absorbed by the composed filter (KS-11 color glass, $2 \mathrm{~mm}$ depth, Russia). The probe beam passes through the pinhole (diameter $\mathrm{mm}$ ) centered at the optical axis and hits the Primary PD photodiode (L-3DP3C, Panasonic, Japan). The signal is converted and amplified by the Control Unit and enters the ADC-DAC unit. Lenses L1 and L2 and the sample compartment can be moved along directions of beams (step, $0.2 \mathrm{~mm}$ ). The spectrometer implements a Secondary PD channel (Fig. 1) for gathering scattering or luminescence signals, if present. An ADC-DAC homemade unit based on C8051F061 board (two 16-bit ADC and two 12-bit DAC channels, ADC time, $2 \mu \mathrm{s}$; readout frequency, $1-5 \mathrm{kHz}$; Silicon Labs, USA) was used in the external-trigger mode from the PC. The homemade software communicates with the unit through a RS-232 interface.

Absorbance measurements in the UV/vis range were carried out using an Agilent Cary 4000 (Australia). A DCS-30 TA Mettler-Toledo instrument (Switzerland) was used to measure specific heats from 288 to $323 \mathrm{~K}$ (scanning rate $10 \mathrm{~K} / \mathrm{min}$; sample mass 20 - $30 \mathrm{mg}$ ). A GRAD 28-35 ultrasound bath (Grad-Technology, Russia) was used. Densities were determined with a VIP-2MP (Russia) vibrating-tube densimeter. Water from a Milli Q water-purification system (Millipore, France) was used: specific resistance $18.2 \mathrm{M} \Omega \times \mathrm{cm}$; dissolved $\mathrm{SiO}_{2} 3 \mathrm{ppb}$; total ion amount $<0.2 \mathrm{ppb}$; TOC $<10 \mathrm{ppb}$; the own thermal-lens signal $0.004 \pm 0.001$. Commercially available 


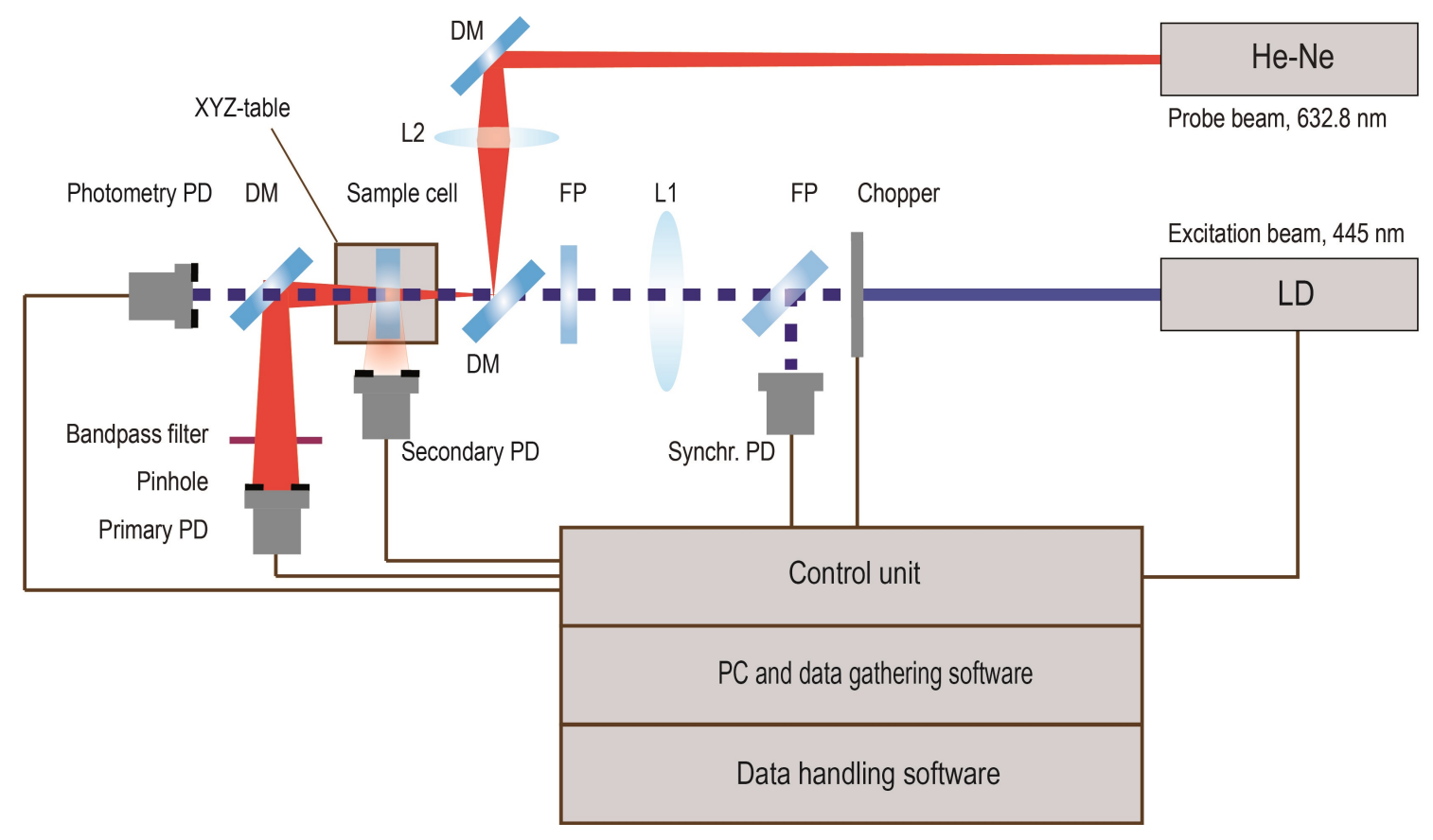

FIG. 1. The scheme of the thermal-lens setup

fullerenes $\mathrm{C}_{60}$ and $\mathrm{C}_{70}, 99.5 \%$ (LLC Neo Tech Product, Russia) were used. The synthesis of $\mathrm{Y} @ \mathrm{C}_{82}$ from fullerene-enriched soot after an arc-discharge synthesis is described previously [4,5]. Unseparated $\mathrm{C}_{78}-\mathrm{C}_{88}$ fractions were obtained by HPLC purification of the soot. AFDs were produced by a standard ultrasound-assisted solvent-exchange procedure. 1.10-phenanthroline iron (II) complex (ferroin sulfate, $0.025 \mathrm{~mol} \cdot \mathrm{L}^{-1}$, Merck) was used as is.

\section{Results and discussion}

The implemented back-synchronized mode of the spectrometer features different measurement conditions for the blooming and dissipating of the transient thermal lens [2]. The advantage is the possibility of transient and steady-state measurements within a single set of experiments. Also, thermal-lens spectrometers usually have a tightly focused excitation beam (beam waist size is $c a$. $50-60 \mu \mathrm{m}[2,6]$ ), which gives a sufficient heating, and, therefore, high sensitivity of optical detection. However, such schematic is not well-suited for studying the thermophysical parameters of disperse systems because for the samples with different thermal conductivities, the heat flows from a small laser-heated zone differ. Hence, the size of the thermal lens would also be different, thus affecting the sensitivity and precision. Thus, for each sample, it would be necessary to change the geometry of the optical scheme to match the sizes of the thermal lens and probe beam. To overcome this, we designed a spectrometer with a wide excitation beam (waist size $300 \mu \mathrm{m}$ ) rendering longer times for attaining a thermal equilibrium.

Despite the aim of thermophysical estimation, we achieved rather low detection limits (by $1-2$ orders of magnitude lower than for photometry). The LOD for ferroin as a model system is $300 \mathrm{nM}$ (at $400 \mathrm{~mW}$ excitation), which is an order of magnitude higher than for classical TLS setups [2], the linear range is slightly wider than two orders of magnitude (the corresponding range of absorptivities for $10 \mathrm{~mm}$ optical paths is $1 \cdot 10^{-5}$ to $2 \cdot 10^{-3} \mathrm{~cm}^{-1}$ ). The local increase in temperature due to the thermal-lens effect is $0.01-20{ }^{\circ} \mathrm{C}$. LODs for fullerenes are $100 \mathrm{nM}$ for $\mathrm{C}_{60}, 80 \mathrm{nM}$ for $\mathrm{C}_{70}$ and $\mathrm{C}_{78}-\mathrm{C}_{88}$, and $60 \mathrm{nM}$ for $\mathrm{Y} @ \mathrm{C}_{82}$, which correlate with absorptivities of ferroin and fullerenes at the working wavelength. The spectrometer has a response time of $0.05-200 \mathrm{~s}$.

Thermal diffusivity was measured from transient thermal-lens curves by the previously reported approach [3]. The transient curves at the time of attainment of the thermal equilibrium for $10^{-6}-10^{-5} \mathrm{M}$ of all the studied fullerenes are fit with the theoretical equations well, and we used the final part of transient curves for estimating the bulk thermophysical properties of AFDs at the thermal equilibrium. The thermal diffusivity values $(1.43 \pm 0.03) 10^{-7} \mathrm{~m}^{2} \mathrm{~s}^{-1}$; thermal effusivities $(1.55 \pm 0.04) 10^{2} \mathrm{Jm}^{-2} \mathrm{~K}^{-1} \mathrm{~s}^{-1 / 2}$, and thermal conductivities for AFDs for all the studied fullerenes from thermal-lens data (with heat capacity and density obtained from other methods) show a negligible change compared to water, which is in rather good concordance with the previous 
papers on fullerenes solubilized in water [7,8]. This approach can be expanded using a more detailed characterization by the deconstruction of transient thermal-lens curves [3], which could provide estimation for thermophysical properties of the dispersed phase, which would be useful for characterizing carbon nanoparticles.

\section{Conclusions}

Thus, we designed the thermal-lens spectrometer with a wide excitation beam, which is suitable for simultaneous determination of substances by optical absorption and the estimation of thermophysical parameters of solutions. Suitable precision of measurements of thermophysical parameters of aqueous fullerene dispersions including metallofullerenes - thermal diffusivity, thermal effusivity, and thermal conductivity - was obtained.

\section{Acknowledgements}

The work is supported by The Russian Science Foundation, grant no. 14-23-00012-P. We are grateful to Dr. I. E. Kareev (Institute of Problems of Chemical Physics of the Russian Academy of Sciences, Chernogolovka, Russia) for the support of endofullerenes and higher fullerene fractions.

\section{References}

[1] Vargas H., Miranda L. Photothermal techniques applied to thermophysical properties measurements (plenary). Rev. Sci. Instrum., 2003, 74 (1), P. 794-799.

[2] Proskurnin M.A., Volkov D.S., et al. Advances in thermal lens spectrometry. J. Anal. Chem., 2015 , 70 (3), P. $249-276$ (in Russian).

[3] Mikheev I.V., Usoltseva L.O., et al. Approach to the Assessment of Size-Dependent Thermal Properties of Disperse Solutions: TimeResolved Photothermal Lensing of Aqueous Pristine Fullerenes C60 and C70. J. Phys. Chem. C, 2016, 120, P. $28270-28287$.

[4] Bubnov V.P., Laukhina E.E., et al. Endohedral Metallofullerenes: A Convenient Gram-Scale Preparation. Chem. Mater., 2002 , 14 (3), P. $1004-1008$

[5] Kareev I.E., Laukhina E., et al. Harnessing Electron Transfer from the Perchlorotriphenylmethide Anion to Y@C82(C2v) to Engineer an Endometallofullerene-Based Salt. Chemphyschem, 2013, 14 (8), P. 1670-1675.

[6] Zharov V.P. Ultrasharp nonlinear photothermal and photoacoustic resonances and holes beyond the spectral limit. Nat. Photonics, 2011, 5 (2), P. 110-116.

[7] Hwang Y., Lee J., et al. Stability and thermal conductivity characteristics of nanofluids. Thermochimica Acta, 2007 , 455 (1), P. 70-74.

[8] Hwang Y., Park H., Lee J., Jung W. Thermal conductivity and lubrication characteristics of nanofluids. Curr. Appl. Phys., 2006, 6, P. 67-71. 